\title{
SITUATION DES PROGRAMMES MIGRATEURS SUR L'ENSEMBLE DES BASSINS VERSANTS GARONNE ET DORDOGNE.
}

\author{
S. BOYER (1), O. GUERRI (2), G. PUSTELNIK (2)
}

(1) MI.GA.DO., Association Migrateurs GAronne DOrdogne, 14 rue de la République, 47240 Bon Encontre, France.

(2) E.P.I.DOR., Etablissement Public Interdépartemental DORdogne, B.P. 13, 24250 Castelnaud-la-Chapelle, France.

\section{RÉSUMÉ}

L'ensemble des bassins Garonne et Dordogne couvre un territoire d'environ $83000 \mathrm{~km}^{2}$. Comme dans la plupart des systèmes hydrographiques européens, un fort recul des populations a été observé au cours du dernier siècle. Les principales causes en sont les aménagements, l'équipement industriel des cours d'eau, l'apparition de pollutions urbaines et industrielles et dans certains cas la surpêche. En 1978, sous l'impulsion du Ministère de l'Environnement, les premières opérations coordonnées de restauration des poissons migrateurs démarrent sur les bassins Garonne et Dordogne avec un «plan saumon ". A cette époque, sept des huit espèces de grands migrateurs amphihalins sont encore présentes : l'esturgeon européen (Acipenser sturio), la grande alose (Alosa alosa), l'alose feinte (Alosa fallax), l'anguille (Anguilla anguilla), la lamproie fluviatile (Lampetra fluviatilis), la lamproie marine (Petromyzon marinus) et la truite de mer (Salmo trutta). Seul le saumon atlantique (Salmo salar) a définitivement disparu.

En 1999, le bilan des plans de restauration met en évidence les avancées suivantes. Au niveau réglementaire, la protection d'une partie des habitats de reproduction est assurée pour plusieurs espèces et la pêche de trois espèces menacées (l'esturgeon, le saumon et la truite de mer) est interdite. Au niveau aménagement, la libre circulation est rétablie sur une grande partie de la Dordogne, de la Vézère, de la Garonne, de l'Ariège, du Tarn et de l'Aveyron, rendant ainsi accessibles les zones de reproduction situées dans le haut bassin. Au niveau biologique, la restauration d'une souche de saumon atlantique est engagée permettant d'envisager un réensemencement sur le long terme (centre de reconditionnement, piscicultures domaniales). Au niveau du suivi des populations, des stations de contrôle sont implantées sur quelques points stratégiques des bassins versants. Les premiers résultats ont montré une colonisation rapide et massive de la grande alose et une installation progressive d'une population de grands salmonidés sur les deux axes Garonne et Dordogne. Les suivis de population laissent toutefois d'importantes incertitudes sur l'évaluation des stocks et les données restent insuffisantes pour apprécier la dynamique de chacune des populations.

Mots-clés : poissons migrateurs, gestion, protection, restauration, programmes, évaluation, populations, suivi. 


\section{REPORT ON THE PROGRAMMES FOR MIGRATORY FISH THROUGHOUT THE GARONNE AND DORDOGNE BASINS.}

\section{ABSTRACT}

The Garonne and Dordogne basins cover an area of about 83,000 square kilometres. As in most of Europe's hydrographic systems, a sharp drop in these fish populations has been noted over the past century. The principal causes have been conflicting management needs, industrial use of the water courses, the appearance of urban and industrial pollution, and, in certain cases, over-fishing. In 1978, at the prompting of the Environment Ministry, the first co-ordinated operations for the restoration of migratory fish got under way in the Garonne and Dordogne basins with a "Salmon Plan ". At that period, seven of the eight large migratory species were still present : the European sturgeon (Acipenser sturio), allis shad (Alosa alosa), twaite shad (Alosa fallax), the common eel (Anguilla anguilla), the river lamprey (Lampetra fluviatilis), sea lamprey (Petromyzon marinus) and the sea trout (Salmo trutta). Only the Atlantic salmon (Salmo salar) had completely disappeared.

In 1999, an evaluation of the main features of the restoration plans revealed the following improvements. On the statutory plane, protection of part of their spawning habitats had been assured for several species, and fishing for three threatened species (sturgeon, salmon and sea trout) had been banned. On the water management plane, freedom of passage had been re-established over large stretches of the Rivers Dordogne, Vézère, Garonne, Ariège, Tarn and Aveyron, thus giving migratory fish access to the breeding grounds in the upper waters. On the biological plane, efforts to restore Atlantic salmon stocks had begun, with structural and organisational backing which held out hope of full re-establishment in the long term (including a reconditioning centre and fish farms). As for keeping check on the populations, control points had been set up at several strategic sites within the river basins, providing information about their colonisation. The first population figures had demonstrated a progressive installation of salmonidae in both the Garonne and Dordogne axes. However, the tracks kept on the migratory fish leave certain important questions open as regards evaluation of the stocks and the data are still insufficient to appraise the dynamics of each individual population.

Key-words : migratory fish, management, protection, restoration, programmes, evaluation, populations, assessment.

\section{INTRODUCTION}

L'ensemble des bassins versants Garonne-Dordogne couvre une surface d'environ $83000 \mathrm{~km}^{2}$ dont $55000 \mathrm{~km}^{2}$ pour le bassin de la Garonne (la longueur de l'axe Garonne étant de $600 \mathrm{~km}$ ), $25000 \mathrm{~km}^{2}$ pour le bassin de la Dordogne (la longueur de l'axe Dordogne étant de $475 \mathrm{~km}$ ) et $3000 \mathrm{~km}^{2}$ pour le bassin côtier de l'estuaire de la Gironde, le plus grand estuaire européen avec $625 \mathrm{~km}^{2}$ pour $80 \mathrm{~km}$ de longueur. Le territoire concerné par les opérations "poissons migrateurs " touche douze départements répartis sur quatre régions (Figure 1).

De nombreuses informations témoignent de l'abondance historique des poissons migrateurs sur les bassins de la Garonne et de la Dordogne (PUSTELNIK, 1982), avec par exemple une sculpture préhistorique de saumon à «l'abri du poisson » aux Eyzies (24), l'extrait d'un contrat de louage qui relate l'abondance des poissons migrateurs sur la Dordogne au $x \mid X^{*}$ siècle ainsi que de nombreux documents relatifs au suivi d'anciennes pêcheries depuis le moyen âge... 


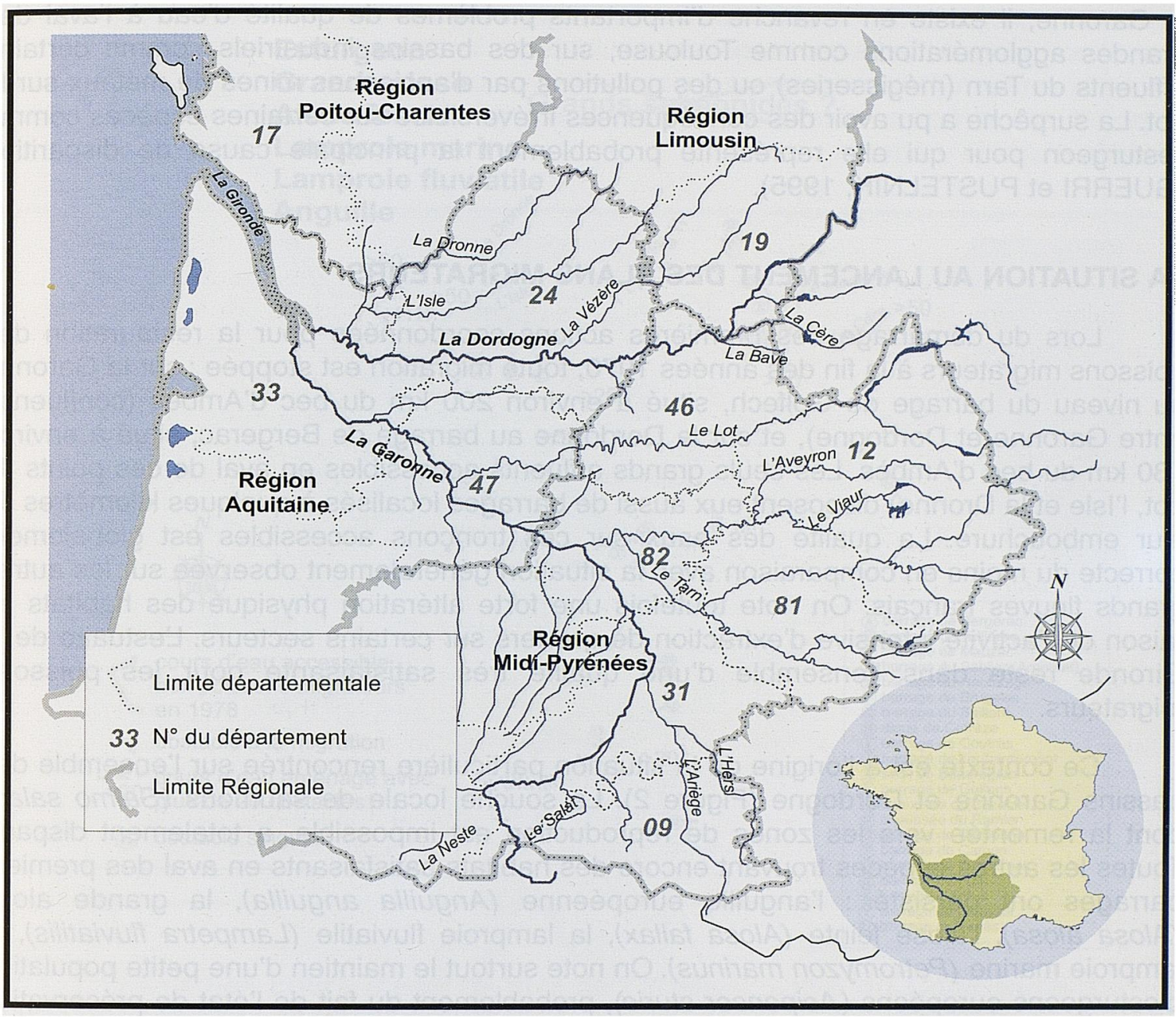

\section{Figure 1}

\section{Carte de situation de l'ensemble des bassins Garonne Dordogne.}

\section{Figure 1}

\section{Geographical situation of the Garonne Dordogne basin.}

A partir de la fin $d u x^{\ominus} x^{\ominus}$ siècle on constate un fort recul des populations de poissons migrateurs sur les bassins Garonne et Dordogne. De la même façon que ce qui a été observé sur la plupart des autres bassins hydrographiques européens, cette régression est due à de multiples causes dont les impacts ont été variables selon les espèces (ROGUET, 1993). Le facteur dominant concerne les obstacles à la migration avec la présence de barrages à Agen (seuil de Beauregard) puis à Golfech et à Toulouse (chaussée du Bazacle) sur la Garonne et à Bergerac, Tuilières et Mauzac sur la Dordogne. Dans l'ensemble, les cours d'eau des deux bassins sont très aménagés, avec de nombreux seuils érigés pour alimenter des moulins (sur la Dronne par exemple) ou pour la navigation (notamment sur l'Isle et le Lot) et de grands barrages à vocation hydroélectrique sur les têtes de bassin de la Dordogne et de la Garonne. L'extraction de granulats est probablement la seconde cause de diminution des stocks de poissons migrateurs avec la destruction importante d'habitats sur deux axes. Cette activité a notamment été très intensive sur la partie basse de ces fleuves. La pollution des eaux n'a probablement pas été déterminante sur le bassin de la Dordogne qui possède peu de grandes agglomérations, peu d'industries et une agriculture peu intensive. Sur le bassin de 
la Garonne, il existe en revanche d'importants problèmes de qualité d'eau à l'aval des grandes agglomérations comme Toulouse, sur des bassins industriels comme certains affluents du Tarn (mégisseries) ou des pollutions par d'anciennes mines de métaux sur le Lot. La surpêche a pu avoir des conséquences irréversibles sur certaines espèces comme l'esturgeon pour qui elle représente probablement la principale cause de disparition (GUERRI et PUSTELNIK, 1995).

\section{LA SITUATION AU LANCEMENT DES PLANS MIGRATEURS}

Lors du démarrage des premières actions coordonnées pour la restauration des poissons migrateurs à la fin des années 1970, toute migration est stoppée : sur la Garonne au niveau du barrage de Golfech, situé à environ $200 \mathrm{~km}$ du bec d'Ambès (confluence entre Garonne et Dordogne), et sur la Dordogne au barrage de Bergerac, situé à environ $130 \mathrm{~km}$ du bec d'Ambès. Les seuls grands affluents accessibles en aval de ces points (le Lot, I'Isle et la Dronne) disposent eux aussi de barrages localisés à quelques kilomètres de leur embouchure. La qualité des eaux sur ces tronçons accessibles est globalement correcte du moins en comparaison avec la situation généralement observée sur les autres grands fleuves français. On note toutefois une forte altération physique des habitats en raison de l'activité intensive d'extraction de graviers sur certains secteurs. L'estuaire de la Gironde reste dans l'ensemble d'une qualité très satisfaisante pour les poissons migrateurs.

Ce contexte est à l'origine de la situation particulière rencontrée sur l'ensemble des bassins Garonne et Dordogne (Figure 2). La souche locale de saumons (Salmo salar), dont la remontée vers les zones de reproduction est impossible, a totalement disparu. Toutes les autres espèces trouvant encore des habitats satisfaisants en aval des premiers barrages ont persisté : l'anguille européenne (Anguilla anguilla), la grande alose (Alosa alosa), l'alose feinte (Alosa fallax), la lamproie fluviatile (Lampetra fluviatilis), la lamproie marine (Petromyzon marinus). On note surtout le maintien d'une petite population d'esturgeons européens (Acipenser sturio), probablement du fait de l'état de préservation exceptionnel de l'estuaire. Quelques salmonidés sont toutefois observés de façon occasionnelle en aval des premiers barrages, et témoignent probablement de la présence de rares individus de truite de mer (Salmo trutta), bloqués au moment de la remontée vers les frayères.

Cette situation permet de comprendre l'importance et la difficulté des programmes de restauration qu'il y avait lieu de mettre en place et surtout la démarche avec laquelle ils ont été initiés. "Ces programmes n'ont pas été lancés avec l'objectif de réaliser une situation primitive d'abondance supposée, compte tenu de l'anthropisation du milieu, mais dans le but de participer à la conservation des espèces et de la biodiversité, de restaurer un patrimoine collectif et de maintenir les usages associés" (POULAIN, Mission Technique Poissons Migrateurs, comm. Pers.).

\section{LES ACQUIS DE VINGT ANNÉES DE PROGRAMMES MIGRATEURS}

En 1978, le premier programme voit le jour avec le lancement des plans Saumon, suivi dans les années 80 par les programmes « migrateurs » et à partir de 1992 par le Contrat Retour aux Sources (PORCHER, 1992 a), qui progressivement viennent structurer les opérations de restauration des poissons migrateurs sur les deux bassins.

\section{Les aspects réglementaires}

Dès le début du programme, les mesures s'orientent vers la mise en place d'un cadre réglementaire, indispensable et préalable à la mise en ceuvre des opérations techniques. 


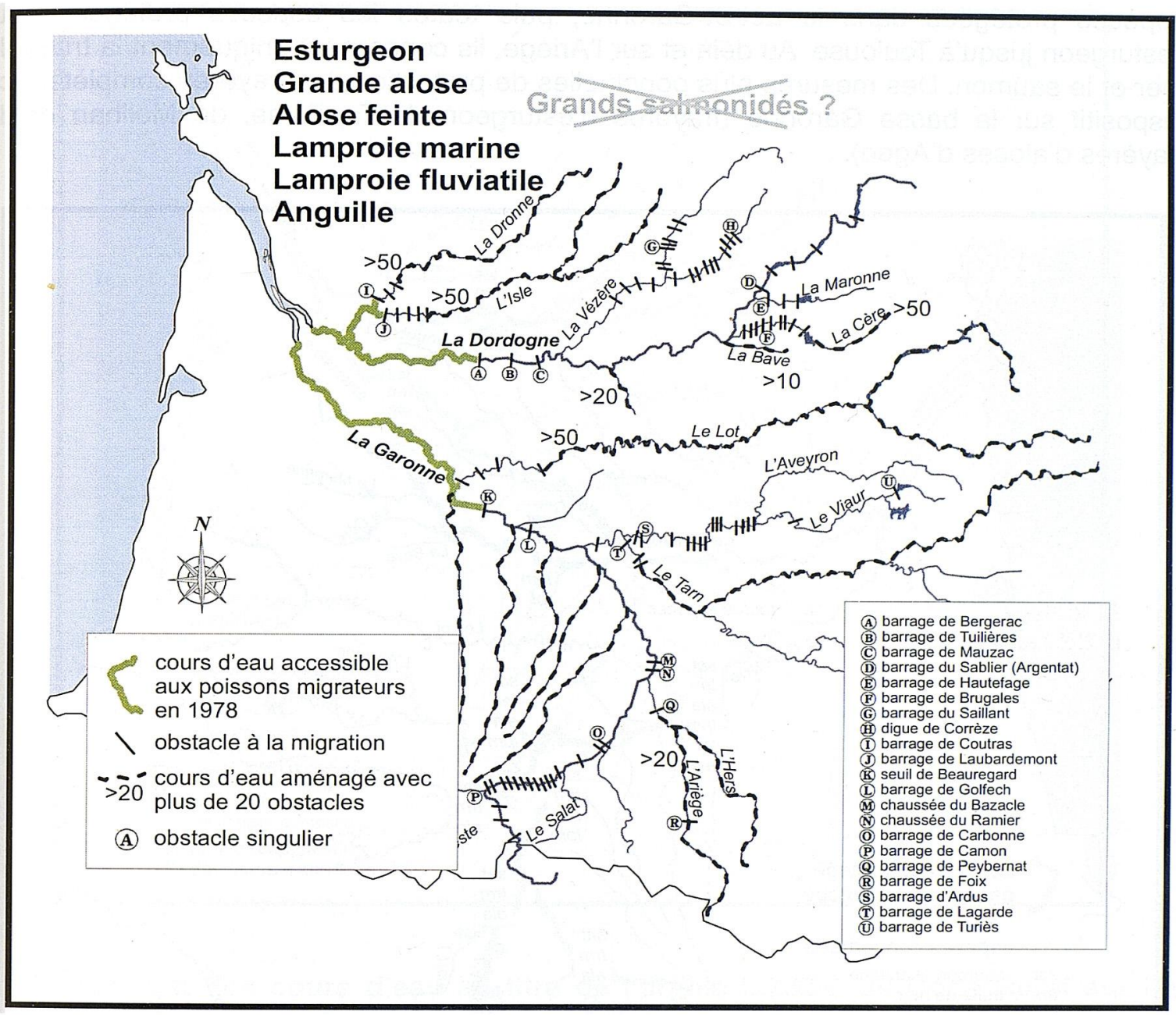

Figure 2

Situation des poissons migrateurs en 1975 sur les bassins Garonne et Dordogne.

\section{Figure 2}

Geographical situation of migratory fish on the Garonne and Dordogne basins in 1975.

\section{Mesures de protection des espèces et des milieux}

En matière de protection des espèces, l'arrêté ministériel du 25/01/1982 protège intégralement l'esturgeon européen et l'arrêté du 08/12/1988, pris en application de la Loi du 10 juillet 1976, fixe la liste des autres espèces de poissons protégés sur l'ensemble du territoire national. Cette liste comprend notamment la lamproie marine, la lamproie fluviatile, le saumon atlantique, la truite de mer, la grande alose et l'alose feinte. En outre, la pêche du saumon et de la truite de mer est interdite sur l'ensemble Garonne, Dordogne et estuaire de la Gironde dès les années 80.

Ce dispositif permet notamment aux Préfets d'arrêter, sur les secteurs concernés, les mesures tendant à favoriser la conservation du biotope dans la mesure où ces derniers sont nécessaires à l'alimentation, la reproduction, au repos ou à la survie des espèces protégées et ce afin de prévenir leur disparition. Largement utilisés sur la rivière Dordogne (Figure 3), ces arrêtés de protection de biotope concernent les 2 aloses, les 2 lamproies, le saumon atlantique dans les départements de la Dordogne, du Lot et de la Corrèze. Sur la Garonne, ils concernent toutes les espèces dans le département de la Gironde, toutes les 
espèces protégées dans le Lot-et-Garonne, puis toutes les espèces protégées sauf l'esturgeon jusqu'à Toulouse. Au delà et sur l'Ariège, ils concernent uniquement la truite de mer et le saumon. Des mesures plus ponctuelles de protection des frayères complètent ce dispositif sur la basse Garonne (frayères d'esturgeon de Tartifume, de Meilhan et de frayères d'aloses d'Agen).

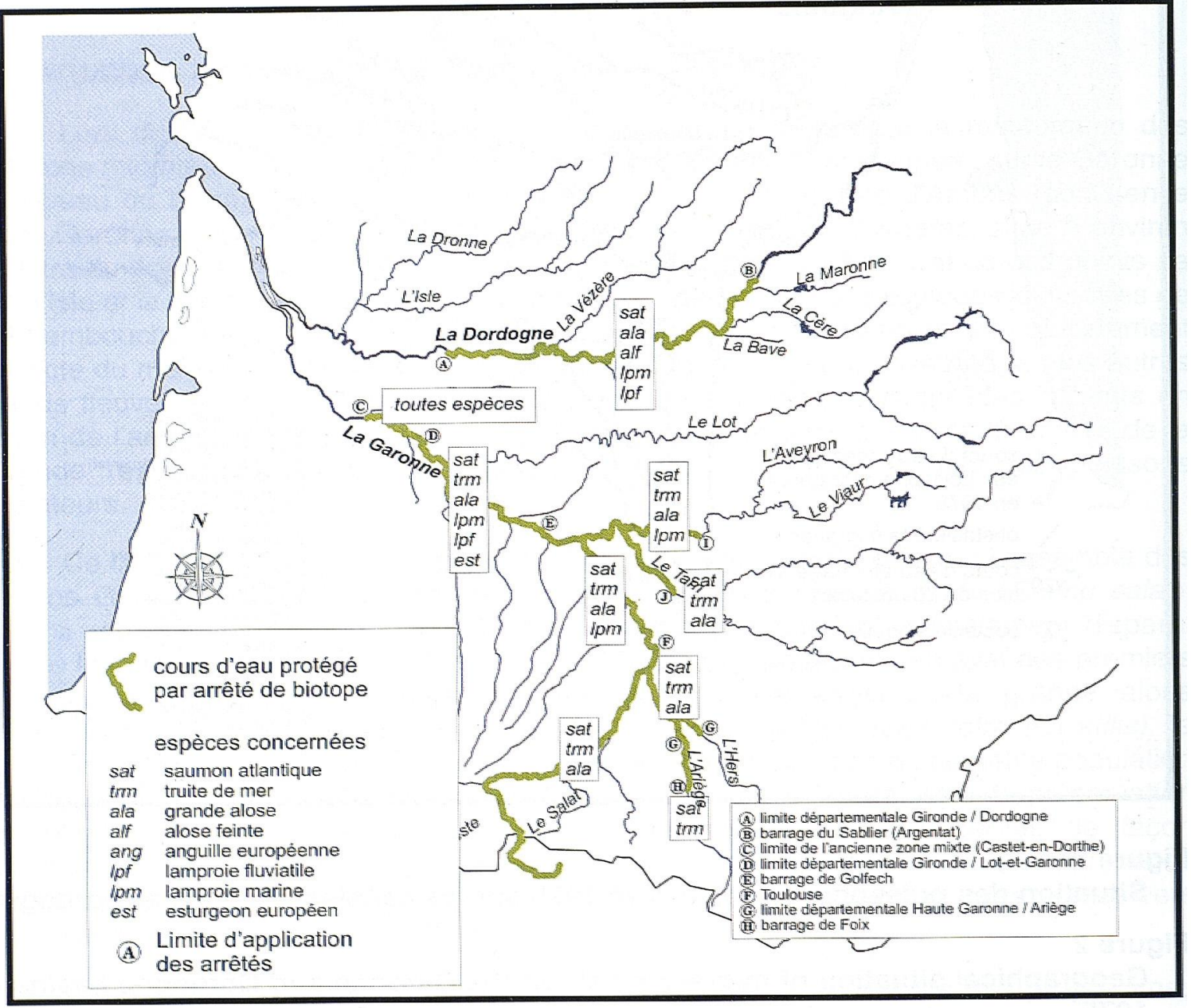

Figure 3

Mesures réglementaires concernant la protection des biotopes des poissons migrateurs sur les bassins Garonne et Dordogne.

\section{Figure 3}

\section{Regulations about protection of migratory fish biotope on the Garonne Dordogne bassin.}

\section{Mesures de restauration des cours deau et de la libre circulation}

Deux types de mesure concernent par ailleurs la restauration des cours d'eau et notamment la reconquête de la libre circulation des poissons migrateurs. Il s'agit d'une part des classements au titre de l'article L.232-6 du Code Rural (application de la Loi Pêche du 29 juin 1984). Ces dispositions donnent obligation de réaliser des dispositifs de franchissement pour les poissons migrateurs, sur des cours d'eau qui correspondent à des parcours de migration classés (arrêté ministériel), et de mettre en conformité les ouvrages existants dans un délai de 5 ans après la publication de la liste des espèces concernees, fixée par arrèté. Sur le bassin de la Dordogne (Figure 4), la Dordogne à laval du barrage du Sablier. la Vézère à l'aval du barrage du Saillant, la Cère à l'aval du barrage de 


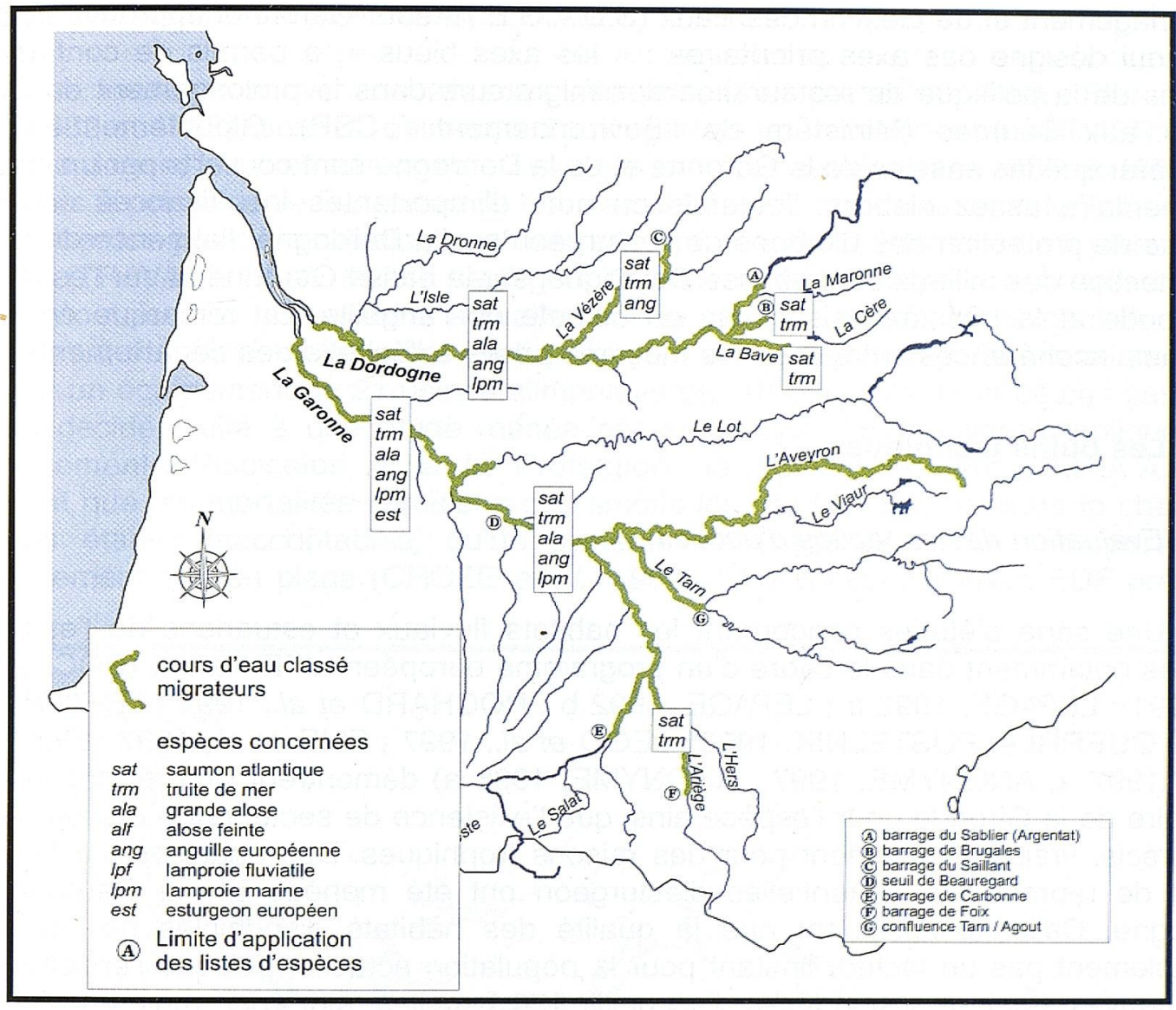

\section{Figure 4}

\section{Classement des cours d'eau au titre de l'article L.232-6 du Code Rural sur les bassins Garonne et Dordogne.}

\section{Figure 4}

\section{Rivers classification according to the L.232-6 article of the French rural code on the Garonne Dordogne bassin.}

Brugales et la Bave sont classées avec une liste d'espèce où figurent les grands migrateurs. Sur le bassin de la Garonne, le classement avec liste d'espèces concerne tout l'axe de la Garonne jusqu'à Carbonne, l'Ariège en aval de Foix, l'Aveyron dans sa totalité et le Tarn jusqu'à sa confluence avec l'Agout. De nombreux autres affluents du bassin sont également classés, mais sans liste d'espèce ou uniquement à l'égard de la truite fario. Un second classement définit des "cours d'eau réservés " qui, au titre de la loi du 15 juillet 1980 sur les économies d'énergie, prévoit que sur certains cours d'eau ou sections de cours dont la liste est fixée par décret en Consell d'Etat, aucune autorisation ou concession ne sera donnée pour des entreprises hydrauliques nouvelles.

\section{Mesures complémentaires}

Des outils complémentaires sont progressivement venus renforcer cet ensemble de mesures réglementaires. D'une part des plans de gestion des poissons migrateurs élaborés par les COmités de GEstion des POissons Migrateurs (décret "amphihalin "du 16 février 1994). Le plan de gestion du bassin Garonne, Dordogne, Charente, Seudre, Leyre, arrêté le 22 décembre 1995, fixe un cadre unique el cohérent pour la gestion des poissons migrateurs sur l'ensemble des eaux continentales du bassin et sur l'estuaire de la Gironde jusqu'à la limite transversale de la mer. D'autre part le Schéma Directeur 
d'Aménagement et de Gestion des Eaux (S.D.A.G.E.) Adour-Garonne, approuvé le 6 août 1996, qui désigne des axes prioritaires : "les axes bleus ", a permis de confirmer les priorités de la politique de restauration des migrateurs dans le prolongement du Contrat Retour aux Sources (Ministère de l'Environnement / CSP). Globalement on peut considérer que les bassins de la Garonne et de la Dordogne sont couverts par un dispositif réglementaire assez élaboré. Toutefois on note d'importantes insuffisances comme le manque de protection des biotopes de l'esturgeon sur la Dordogne, l'absence de mesure de protection des milieux sur la basse Dordogne, sur la basse Garonne et sur l'estuaire de la Gironde et la très mauvaise prise en compte de l'anguille. On remarque également certaines incohérences entre diverses mesures prises à l'échelle des départements.

\section{Les outils techniques}

\section{Évaluation des capacités d'accueil}

Une série d'études concernant les habitats fluviaux et estuariens de l'esturgeon, menées notamment dans le cadre d'un programme européen LIFE-Nature (ROCHARD et al, 1991 ; LEPAGE, 1992 a ; LEPAGE, 1992 b ; ROCHARD et al., 1992 ; LEPAGE et al, 1993 ; GUERRI et PUSTELNIK, 1996 ; JEGO et al., 1997 ; ELIE et al., 1997 ; ROCHARD et al., 1997 a, ANONYME, 1997 ; ANONYME, 1998 a) démontrent l'intérêt particulier de l'estuaire de la Gironde pour l'espèce ainsi que l'existence de secteurs de rassemblement très précis, vraisemblablement pour des raisons trophiques. Des analyses sur l'état des zones de reproduction potentielles d'esturgeon ont été menées sur la Garonne et la Dordogne. Celles-ci concluent que la qualité des habitats disponibles ne constituent probablement pas un facteur limitant pour la population actuelle, puisque l'ensemble des deux fleuves seraient susceptibles d'accueillir 170 femelles, alors que moins de quarante géniteurs migrants ont été observés au cours de vingt dernières années.

Quelques analyses ponctuelles ont été réalisées sur les potentialités des cours d'eau pour la grande alose (PUSTELNIK, 1982 ; CASSOU-LEINS et al., 1988 ; FATIN et DARTIGUELONGUE, 1995 ; MENCHI et MAURIES, 1999), mais la plus grande partie des études menées en matière d'habitats a été ciblée sur le saumon atlantique (TINEL, 1983 ; KERHOAS, 1984 ; BRUZY, 1985 ; GAYOU, 1986 ; ANONYME, 1992 a ; ANONYME, 1992 b ; DARTIGUELONGUE, 1992 ; DULUDE et al, 1992 ; GAYOU, 1993 ; GUIDET, 1994 ; MARCHAT et al., 1994 ; SOULET, 1994). Ces travaux ont permis de valider la qualité des habitats disponibles par cours d'eau et d'évaluer les capacités d'accueil de ceux-ci en vue d'opérations de repeuplement pour le saumon. Ainsi pour la Garonne et ses principaux affluents en amont de Toulouse (Garonne amont Montréjeau, Pique, Neste, Salat, Ariège, Vicdessos et Hers), $663 \mathrm{~km}$ de cours d'eau ont été décrits et cartographiés. Actuellement la surface retenue est estimée à 340 ha d'équivalents-radiers/rapides, ce qui représente une capacité d'accueil théorique en smolts sauvages de 166600 équivalents smolts (sur la base de 4,9 équivalents-smolts sauvages par $100 \mathrm{~m}^{2}$ ). Sur la Dordogne et ses principaux affluents (Dordogne à l'aval du barrage du Sablier, Maronne à l'aval du barrage de Hautefage, Bave, Vézère à l'aval du barrage du Saillant et Corrèze aval de la digue de Corrèze) les surfaces théoriques de grossissement sont évaluées à 345 ha, ce qui représente une capacité d'accueil de 103200 équivalents smolts sauvages (sur la base de 3 équivalents-smolts sauvages par $100 \mathrm{~m}^{2}$ ) (DULUDE et al, 1997). Il est noté que le nombre d'équivalents-smolts sauvages correspondant, d'après PORCHER (1992), à environ 3 ou 4 saumoneaux ou smolts issus d'une production en élevage, c'est par un facteur moyen de 3,5 qu'il convient de multiplier les besoins en terme de repeuplement. Ces analyses ont guidé les décisions d'engager, une fois les possibilités de restauration globalement confirmées, les grandes actions d'équipement des cours d'eau pour la montaison et les opérations de repeuplement. 


\section{Dispositifs de franchissement}

Des mesures conventionnelles ont été prises, en particulier entre l'Etat et EDF (entre 1982 et 1988), pour la réalisation d'ouvrages de franchissement (LARINIER, 1993). Sur la Dordogne, les premiers grands ouvrages ont été réalisés entre 1985 et 1990 avec les passes à poissons de Bergerac, de Mauzac et l'ascenseur à poissons de Tuilières. Sur la Garonne, les premières réalisations ont concerné, en 1987, l'ascenseur à poissons de Golfech, (TRAVADE et al., 1996). La Figure 5 présente les principaux ouvrages équipés pour la montaison et les secteurs actuellement accessibles. Le rétablissement de la libre circulation a été traité de manière particulière sur un secteur de la Garonne amont qui présente un équipement de 21 usines alimentées par 18 barrages. Pour ce cas particulier il a été décidé, suite à une étude menée par le Groupe d'Hydraulique Appliquée aux Aménagements Piscicoles et à la Protection de l'Environnement (G.H.A.A.P.P.E.) concluant que les mortalités cumulées des smolts lors de leur traversée de la chaîne de barrages étaient inacceptables, qu'un système de piégeage et de transport serait provisoirement mis en place (CROZE et al., 1998). Une convention avec EDF prévoit, à

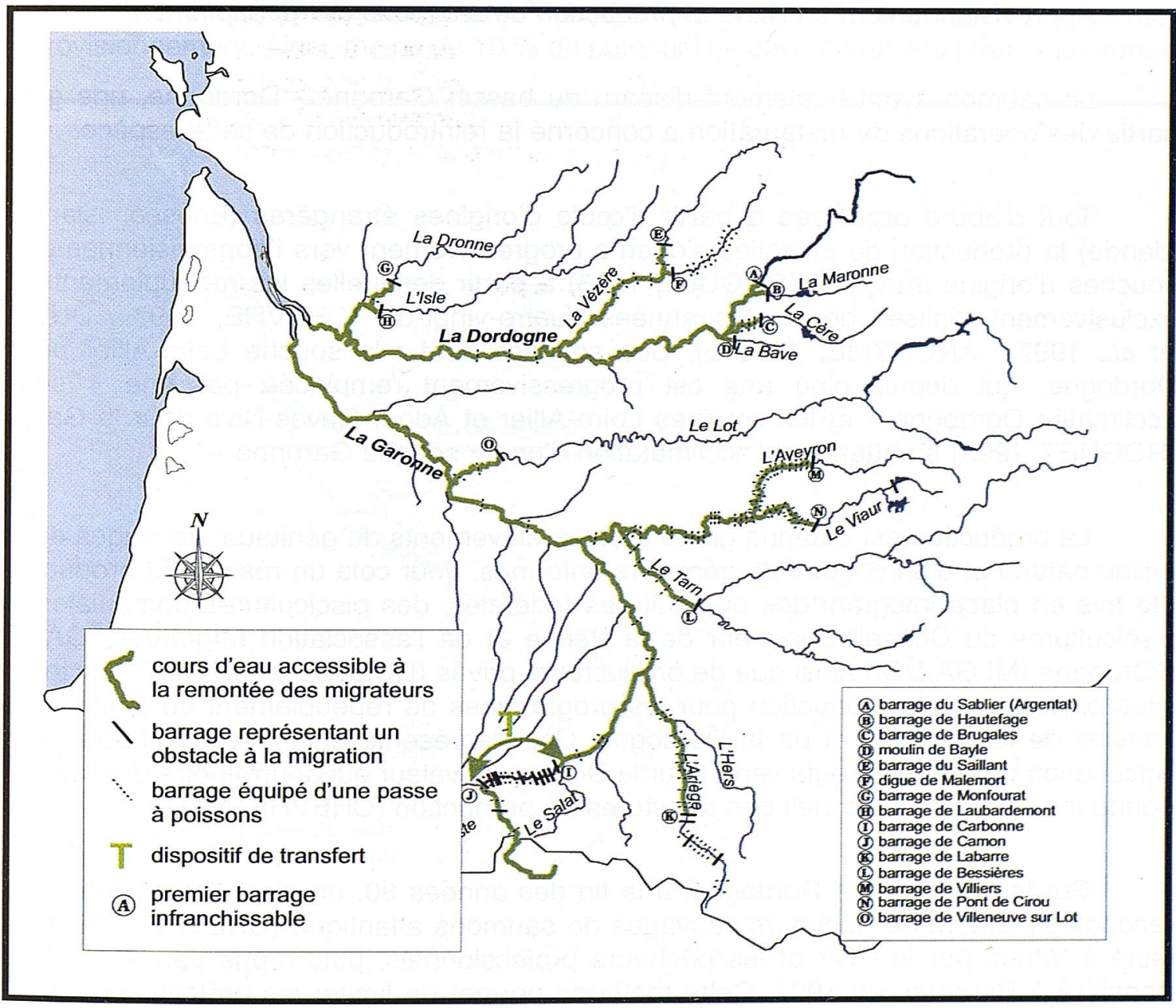

\section{Figure 5}

Cours d'eau accessibles et barrages équipés d'un système de franchissement pour les poissons migrateurs sur les bassins Garonne et Dordogne.

\section{Figure 5}

Rivers accessibility and dams with fish facilities for the migratory fish on the Garonne Dordogne bassin. 
partir de deux stations de piégeage, la capture des salmonidés juvéniles lors de leur dévalaison et des géniteurs lors de leur migration génésique. Les juvéniles doivent ensuite être transférés, à l'aide de véhicules spécialement équipés, à l'aval du dernier barrage non aménagé de la chaîne, et les géniteurs l'amont du dernier barrage non aménagé de la chaîne.

L'ensemble des aménagements réalisés sur les principaux axes de migration ont été calibrés pour le passage des aloses, espèce la plus exigeante en matière de franchissement (LARINIER et TRAVADE, 1992). Ils ont ainsi profité à l'ensemble des espèces, à l'exception de l'anguille, qui aux stades civelle et anguillette, nécessite des aménagements spécifiques, adaptés à la taille des individus migrants (PORCHER, 1992 b). Actuellement, peu de dispositifs existent pour cette espèce mais des études ont été engagées depuis 1997, sur le site de Tuilières, pour tester un dispositif de franchissement adapté aux différentes écophases (DARTIGUELONGUE, TRAVADE et PALLO, en projet).

\section{Approvisionnement en cufs et production de saumons de repeuplement}

Le saumon ayant totalement disparu du bassin Garonne - Dordogne, une grande partie des opérations de restauration a concerné la réintroduction de cette espèce.

Tout d'abord organisée à partir d'cufs d'origines étrangères (Ecosse, Islande et Irlande) la production de juvéniles s'oriente progressivement vers l'approvisionnement en souches d'origine française (ROGUET, 1993) à partir desquelles les repeuplements sont exclusivement réalisés depuis les années quatre-vingt-dix (CHEVRE, 1997 ; DULUDE et al, 1997 ; ANONYME, 1998 b). Ces souches sont : la souche Loire-Allier pour la Dordogne, qui depuis cinq ans est progressivement remplacée par une " souche acclimatée Dordogne » et les souches Loire-Allier et Adour-Gaves-Nive pour la Garonne (ROGUET, 1993) en attendant l'acclimatation d'une « souche Garonne ".

La production est obtenue grâce à des prélèvements de géniteurs sauvages dans le milieu naturel et d'un cheptel de géniteurs enfermés. Pour cela un réseau de production a été mis en place intégrant des piscicultures fédérales, des piscicultures domaniales, des piscicultures du Conseil Supérieur de la Pêche et de l'association Mlgrateurs GAronne DOrdogne (MI.GA.DO.) ainsi que de producteurs privés (DULUDE et al. 1997). En tout, 14 sites participent à la production pour les programmes de repeuplement en saumons des bassins de la Garonne et de la Dordogne. Ceci représente en moyenne chaque année l'incubation de 800000 œuís verts pour la Dordogne, valeur qui pourrait être doublée étant donné les capacités d'accueil des structures de production (CHEVRE, 1997).

Sur le bassin de la Dordogne, à la fin des années 80 , un dispositif original pour le reconditionnement de géniteurs sauvages de saumons atlantique (CHEVRE, 1997) a été testé à Vitrac, par le CSP et les pêcheurs professionnels, puis repris par MI.GA.DO. et transféré à Bergerac en 1994. Cette méthode permet de limiter les prélèvements sur les stocks naturels tout en assurant la production pendant plusieurs années d'ceufs d'origine sauvage. Actuellement une centaine de géniteurs maintenus en stabulation permet de produire entre 200 et 300000 œufs embryonnés. Sur le bassin de la Garonne, une filière d'élevage extensif s'est développée vers les années quatre-vingt-dix au niveau des lacs ariégeois de Prat St-Martin et de Moulzoune pour produire des tacons semi-sauvages d'automne. Ce mode d'élevage économique permet le délestage des piscicultures et la production de sujets rustiques bien adaptés au milieu qui chaque année représente en moyenne 26500 tacons O+ d'automne (GAYOU, 1999). 


\section{Déversement de saumons de repeuplement}

Après quelques années d'expérimentation nécessaires à l'organisation de la filière de production, à la mise au point des techniques d'élevage et des méthodes de transport, il a été possible d'augmenter la production de poissons et les déversements. Progressivement, les plans d'alevinages s'affinent et le nombre de secteurs concernés augmente au rythme du rétablissement de leur accessibilité. Initialement seuls les grands axes étaient alevinés, puis les repeuplements en saumon atlantique ont été étendus à un certain nombre d'affluents (Figure 6). Les déversements moyens sont actuellement de l'ordre de 140000 équivalents-smolts d'élevage pour la Dordogne. En comparant ces chiffres au potentiel du bassin de la Dordogne, estimé en équivalents-smolts sauvages, on pourrait considérer que la saturation des habitats disponibles est pratiquement atteinte. Toutefois, ces repeuplements étant réalisés à partir de sujets issus de l'élevage, le nombre de poissons déversés pourrait être majoré. Par ailleurs, l'évaluation du potentiel du bassin de la Dordogne n'est pas complètement exhaustif, certains cours d'eau n'ayant pas encore fait l'objet d'estimation précise. Sur la Garonne après avoir atteint un niveau d'environ 100000 équivalents-smolts d'élevage en 1997 et 1998, les déversements sont retombés à environ 40000 équivalents-smolts d'élevage en 1999, en raison de problèmes d'approvisionnement. Ainsi, moins de $10 \%$ du potentiel global a été atteint pour cette année.

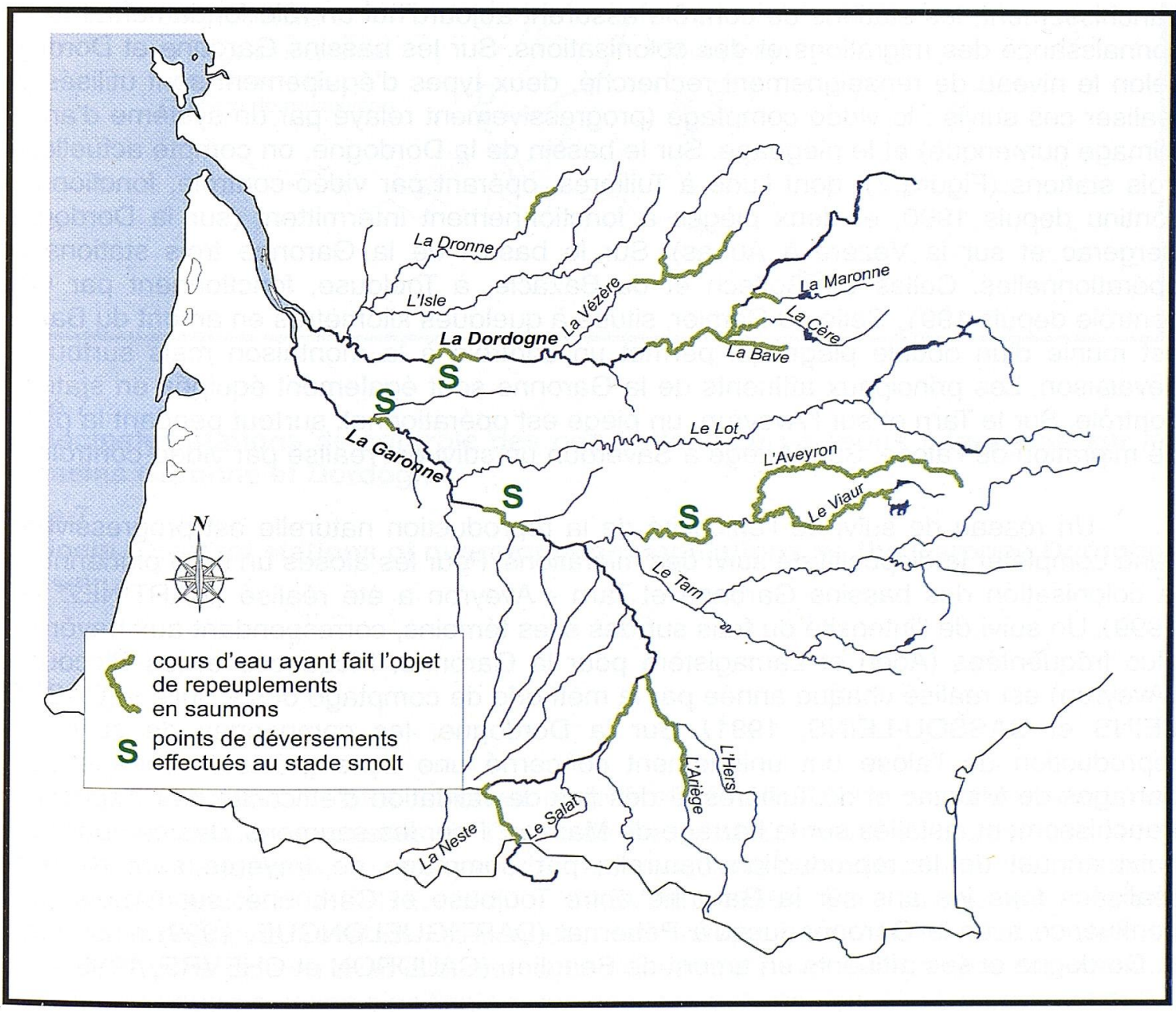

Figure 6

Repeuplements en saumons sur les bassins Garonne et Dordogne.

Figure 6

Stocking areas of the young salmon on the Garonne Dordogne bassin. 


\section{Reconstitution des populations d'esturgeons}

L'esturgeon, en raison du statut de l'espèce, de l'état très dégradé de la population du bassin de la Gironde, et du fait qu'il s'agit de la seule population relique à l'échelle de son aire initiale de répartition, a également fait l'objet d'opérations d'acclimatation à la captivité, en vue de mettre en place une filière de production pour le repeuplement. Ces actions, expérimentées par l'Unité Ressources Aquatiques Continentales du CEMAGREF, dans le cadre du programme LIFE-Nature (BRUN et ROUAULT, 1997 a ; ROUAULT et BRUN, 1997 ; WILLIOT, 1997 a, WILLIOT, 1997 b ; WILLIOT, 1997 c ; ANONYME, 1997), sont destinées à anticiper sur le risque de poursuite de la chute de la populations, qui conduirait à l'extinction de l'espèce, et à constituer la base de futures réimplantations dans d'autres bassins versants autrefois colonisés.

\section{Outils d'évaluation}

Concernant le suivi des populations, les bases d'un réseau ont été installées d'une part à partir de stations fixes de contrôle des migrations et d'autre part, pour le saumon, à partir d'opérations saisonnières d'échantillonnage sur les populations.

Après avoir permis dans un premier temps de vérifier l'efficacité des ouvrages de franchissement, les stations de contrôle assurent aujourd'hui un rôle fondamental dans la connaissance des migrations et des colonisations. Sur les bassins Garonne et Dordogne, selon le niveau de renseignement recherché, deux types d'équipement sont utilisés pour réaliser ces suivis : le vidéo comptage (progressivement relayé par un système d'analyse d'image numérique) et le piégeage. Sur le bassin de la Dordogne, on compte actuellement trois stations (Figure 7), dont l'une à Tuilières, opérant par vidéo-contrôle, fonctionne en continu depuis 1990, et deux pièges à fonctionnement intermittent (sur la Dordogne à Bergerac et sur la Vézère à Aubas). Sur le bassin de la Garonne trois stations sont opérationnelles. Celles de Golfech et du Bazacle, à Toulouse, fonctionnent par vidéocontrôle depuis 1991. Celle du Ramier, située à quelques kilomètres en amont du Bazacle, est munie d'un double piège qui permet un contrôle à la montaison mais surtout à la dévalaison. Les principaux affluents de la Garonne sont également équipés en station de contrôle. Sur le Tarn et sur l'Aveyron, un piège est opérationnel, surtout pendant la période de migration de l'alose. Sur l'Ariège à Saverdun un suivi est réalisé par vidéo-contrôle.

Un réseau de suivi de l'efficacité de la reproduction naturelle est progressivement venu compléter le dispositif de suivi des migrations. Pour les aloses un suivi pluriannuel de la colonisation des bassins Garonne et Tarn - Aveyron a été réalisé (MARTINEZ et al., 1999). Un suivi de l'intensité du frais sur des sites témoins, correspondant aux frayères les plus fréquentées (Agen et Lamagistère pour la Garonne, Picquecos et les Biccous sur l'Aveyron) est réalisé chaque année par la méthode de comptage des «bulls 》 (CASSOULEINS et CASSOU-LEINS, 1981). Sur la Dordogne, les campagnes de suivi de la reproduction de l'alose ont uniquement concerné une zone précise, située entre les barrages de Mauzac et de Tuilières, à des fins de validation d'efficacité des dispositifs de franchissement installés sur le barrage de Mauzac. Pour les saumons, des campagnes de suivi annuel de la reproduction naturelle par comptage de frayères sont désormais réalisées tous les ans sur la Garonne entre Toulouse et Carbonne, sur l'Ariège de sa confluence avec la Garonne jusqu'à Pébernat (DARTIGUELONGUE, 1999) ainsi que sur la Dordogne et ses affluents en amont de Beaulieu (CAUDRON et CHEVRE, 1999).

Un suivi par pêches électriques est effectué pour évaluer l'efficacité des repeuplements en saumons. Réalisées afin d'apporter des informations sur la qualité des zones de production, sur l'abondance des effectifs présents et sur l'état sanitaire des populations, ces campagnes de pêche sont programmées chaque année sur les deux bassins. Les densités retrouvées sur les secteurs amonts de la Dordogne et ses affluents 


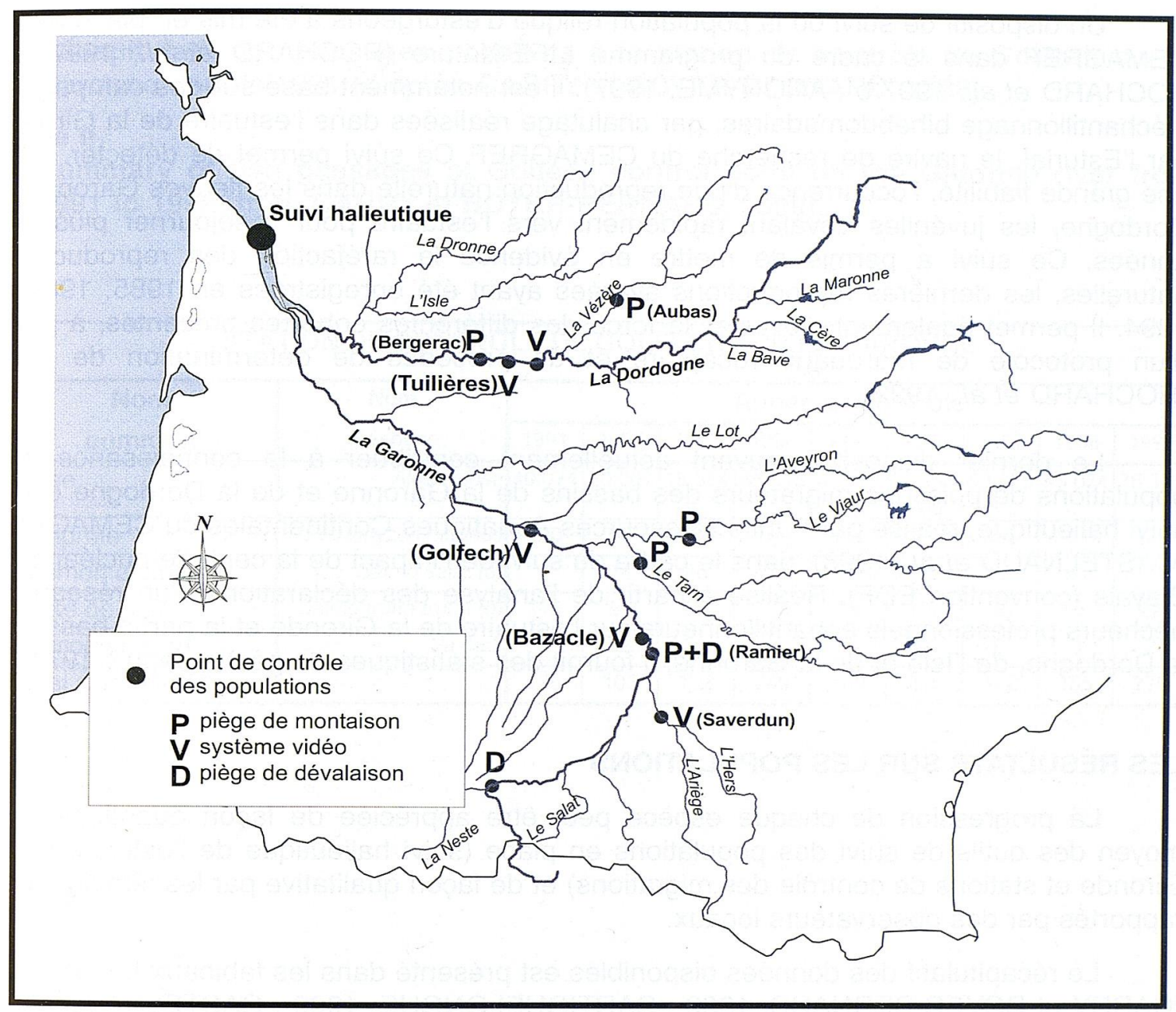

\section{Figure 7}

Principales stations de contrôle des populations de poissons migrateurs sur les bassins Garonne et Dordogne.

\section{Figure 7}

Principal control stations of migratory fish populations on the Garonne Dordogne bassin.

sont bonnes, puisque l'on peut retrouver entre 5 et 25 ind./100 $\mathrm{m}^{2}$ selon la densité des alevinages réalisés. Des densités de l'ordre de 50 ind./100 $\mathrm{m}^{2}$ ont été constatées sur le bassin versant de la Bave, qui semble offrir des conditions particulièrement intéressantes pour les salmonidés (CHEVRE, 1997 ; CHEVRE, 1998). Sur l'Ariège les résultats présentent une grande variabilité stationnelle, les stations amont étant plus productives que celles situées plus en aval. Globalement l'utilisation d'alevins en nombre important se traduit par une augmentation significative des densités automnales. Ainsi, à partir de 1993, le renforcement du repeuplement s'est-il concrétisé par l'apparition de valeurs supérieures à 5 ind. $/ 100 \mathrm{~m}^{2}$, avec de grandes variabilités, de 0.1 à 60 ind. $/ 100 \mathrm{~m}^{2}$, d'une station à l'autre. Ces variations traduisent à la fois une évolution spatiale de la répartition des habitats à haut rendement et une variation dans le temps de la capacité d'accueil de certaines stations (GAYOU, 1999). 
Un dispositif de suivi de la population relique d'esturgeons a été mis en place par le CEMAGREF dans le cadre du programme LIFE-Nature (ROCHARD et al., 1997 a ; ROCHARD et al., 1997 b ; ANONYME, 1997). Il est notamment basé sur des campagnes d'échantillonnage bihebdomadaires, par chalutage réalisées dans l'estuaire de la Gironde par l'Esturial, le navire de recherche du CEMAGREF. Ce suivi permet de détecter, avec une grande fiabilité, l'occurrence d'une reproduction naturelle dans les fleuves Garonne et Dordogne, les juvéniles dévalant rapidement vers l'estuaire pour y séjourner plusieurs années. Ce suivi a permis de mettre en évidence la raréfaction des reproductions naturelles, les dernières reproductions avérées ayant été enregistrées en 1985, 1988 et 1994. II permet également d'évaluer la force des différentes cohortes présentes, à partir d'un protocole de marquage recapture et de procédés de détermination de l'âge (ROCHARD ef al., 1992).

Le dernier dispositif pouvant actuellement contribuer à la connaissance des populations de poissons migrateurs des bassins de la Garonne et de la Dordogne est le suivi halieutique, réalisé par l'Unité Ressources Aquatiques Continentales du CEMAGREF (CASTELNAUD et al., 1998), dans le cadre du suivi de l'impact de la centrale nucléaire du Blayais (convention EDF). Réalisé à partir de l'analyse des déclarations d'un réseau de pêcheurs professionnels échantillonneurs sur l'estuaire de la Gironde et la partie basse de la Dordogne, de l'Isle et de la Garonne, il fournit des statistiques de pêche depuis 1978.

\section{LES RÉSULTATS SUR LES POPULATIONS}

La progression de chaque espèce peut ère appréciée de taçon quantiltative au moyen des outils de suivi des populations en place (suivi halieutique de l'estuaire de la Gironde et stations de contrôle des migrations) et de façon qualitative par les têmoignages rapportés par des observateurs locaux.

Le récapitulatif des données disponibles est présenté dans les tableaux I, II, III et IV (CARRY et BOYER-BERNARD, 1999 ; DARTIGUELONGUE, 1999 ; CASTELNAUD et aV, 1998). Etant donnẻe la position des stations de contrôle sur les bassins, les effectífs de migration doivent être interprêtés en fonction de l'espèce considérëe, de la présence ou non de frayères à l'aval, et des conditions hydrochimatiques de lannée. La déclinaison des résultats par espèce permet globalement de constater les situations suivantes.

\section{Tableau I}

Récapitulatif des passages observés à la station de contrôle de Tullières sur la Dordogne depuis 1991 (d'après DARTIGUELONGUE et LANGON, 1999).

\section{Table 1}

Summary of fish passages at Tuillières control point on the Dordogne fiver firom 1991 to 1999 (from DARTIGUELONGUE et LANGON, 1999).

\begin{tabular}{|c|c|c|c|c|c|c|c|c|c|c|}
\hline \multicolumn{11}{|c|}{ STATION DE CONTROLE DE TULUERES SUR LL DORDOGIE } \\
\hline \multirow{2}{*}{$\frac{\text { Nom }}{\text { commun }}$} & \multirow{2}{*}{$\frac{\text { Nom }}{\text { d"especes }}$} & \multicolumn{9}{|c|}{ Année de contróle } \\
\hline & & $\pi 991$ & 1992 & 7993 & 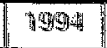 & 1995 & 7996 & 1907 & $\operatorname{19998}$ & 71999 \\
\hline Alose & AnO $: 105$ & 6053 & 11945 & 39704 & 152592 & 72.245 & 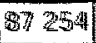 & 42374 & 284465 & 163300 \\
\hline Angullle juvenille & Anp & 73496 & 16307 & $7+592$ & 113.344 & 3207 & 5035 & 20608 & 866 & 71184 \\
\hline Lampiole & 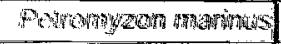 & 7350 & 9009 & 16992 & 4368 & 4559 & 2923 & 29513 & 4225 & 4567 \\
\hline Saumon atlantigue & 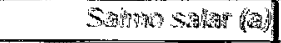 & 9 & 82 & 8 & 332 & 98 & $29 \sqrt{36}$ & 528 & 795 & 484 \\
\hline Trute de mer & 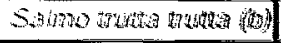 & 4 & 23 & 297 & 305 & 93 & 165 & 275 & 95 & 123 \\
\hline Salmomaldes mol. & 8 & 7 & 67 & 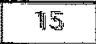 & $\pi$ & $\pi$ & (0) & $\overline{0}$ & (0) & (1) \\
\hline Total salmonides & & 8 & 166 & 3is & 1638 & 192 & 461 & $80 \pi$ & 2990 & 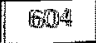 \\
\hline
\end{tabular}




\section{Tableau II}

Récapitulatif des passages observés à la station de contrôle de Golfech sur la Garonne depuis 1991 (d'après CARRY et BOYER-BERNARD, 1999).

Table II

Summary of fish passages at Golfech control point on the Garonne river from 1991 to 1999 (from CARRY et BOYER-BERNARD, 1999).

\begin{tabular}{|c|c|c|c|c|c|c|c|c|c|c|}
\hline \multicolumn{11}{|c|}{ STATION DE CONTRÓLE DE GOLFECH SUR LA GARONNE } \\
\hline \multirow{2}{*}{$\begin{array}{c}\text { Nom } \\
\text { commun }\end{array}$} & \multirow{2}{*}{$\begin{array}{c}\text { Nom } \\
\text { d'espèce }\end{array}$} & \multicolumn{9}{|c|}{ Année de contrôle } \\
\hline & & 1991 & 1992 & 1993 & 1994 & 1995 & 1996 & 1997 & 1998 & 1999 \\
\hline Alose & Alosa alosa & 40074 & 20007 & 18554 & 85813 & 85624 & $\begin{array}{l}106 \\
706\end{array}$ & 98.819 & 49.074 & 36373 \\
\hline Lamproie & Petromyzon marinus & 200 & 747 & 2086 & 107 & 741 & 2382 & 663 & 1678 & 222 \\
\hline Saumon atlantique & Salmo salar (a) & 7 & 14 & 46 & 134 & 118 & 115 & 62 & 90 & 255 \\
\hline Truite de mer & Salmo trutta trutta $(b)$ & 116 & 57 & 55 & 109 & 68 & 108 & 60 & 65 & 22 \\
\hline Salmonidés ind. & a oub & 3 & 32 & 31 & 6 & 3 & 3 & 0 & 0 & 2 \\
\hline Total salmonidés & & 126 & 103 & 132 & 249 & 189 & 226 & 122 & 155 & 273 \\
\hline
\end{tabular}

Tableau III

Récapitulatif des passages observés à la station de contrôle du Bazacle sur la Garonne depuis 1991 (d'après DARTIGUELONGUE et LANGON, 1999).

Table III

Summary of fish passages at Bazacle control point on the Garonne river from 1991 to 1999 (from DARTIGUELONGUE et LANGON, 1999).

\begin{tabular}{|c|c|c|c|c|c|c|c|c|c|c|}
\hline \multicolumn{11}{|c|}{ STATION DE CONTRÓLE DU BAZACLE SUR LA GARONNE } \\
\hline \multirow{2}{*}{$\begin{array}{c}\text { Nom } \\
\text { commun }\end{array}$} & \multirow{2}{*}{$\begin{array}{c}\text { Nom } \\
\text { d'espèce }\end{array}$} & \multicolumn{9}{|c|}{ Année de contrôle } \\
\hline & & 1981 & 1982 & 1993 & 1994 & 1995 & 1996 & 1997 & 1998 & 1999 \\
\hline Aloses & Alosa alosa & 6715 & 1755 & 3765 & 8010 & 20546 & 20279 & 16329 & 4.554 & $38 \pi$ \\
\hline Anguille juwérille & Anguilla anguila & 3 & $\pi$ & 19 & 19 & 3 & $\mathbb{8}$ & 57 & 12 & 1 \\
\hline Anguille adulte & Anguinta anguilla & 60 & $\pi$ & 3 & 0 & 3 & 2 & 15 & 0 & (1) \\
\hline Lamproie & Petromyzon marimus & 24 & 33 & 652 & 4 & $B 4$ & 591 & 40 & 268 & 30 \\
\hline Saumon atlantique & Salmo salar (a) & 5 & 5 & 21 & 55 & 37 & 6 & 10 & 37 & 46 \\
\hline Trutte de mer & Sakno butta trutta (b) & 22 & 8 & 49 & 54 & 53 & 49 & 34 & 27 & 49 \\
\hline Salmonides ind. & a ou $\mathrm{b}$ & 4 & 0 & $\pi$ & 8 & 0 & 8 & 0 & D & 0 \\
\hline Total salmonidés & & 31 & 13 & 71 & $\pi 17$ & 90 & 110 & 44 & 64 & geg \\
\hline
\end{tabular}




\section{Tableau IV}

Evolution des productions de la pêche professionnelle dans le système fluvioestuarien Gironde Garonne Dordogne entre 1978 et 1996 (en tonnes). *Valeurs très nettement surestimées. D'après CASTELNAUD et al. (1998).

\section{Table IV}

ODordogne from 1978 to 1996 (in tons ). *Values largely overestimed. From CASTELNAUD and al. (1998).

\begin{tabular}{|c|c|c|c|c|c|c|c|c|c|c|c|c|c|c|}
\hline \multirow{3}{*}{$\begin{array}{c}\text { Nom } \\
\text { de l'espèce }\end{array}$} & \multicolumn{14}{|c|}{ SUIVI HALIEUTIQUE DE L'ESTUAIRE DE LA GIRONDE } \\
\hline & \multicolumn{14}{|c|}{ Année de contrôle } \\
\hline & 1978 & 1981 & 1982 & 1983 & 1984 & 1988 & 1989 & 1990 & 1991 & 1992 & 1993 & 1994 & 1995 & 1996 \\
\hline $\begin{array}{l}\text { Alose vraie } \\
\text { (Alosa alosa) }\end{array}$ & 500 & 826 & 863 & 1096 & 615 & 1007 & 762 & 487.2 & 840.6 & 480 & 338 & 500 & 577 & 539 \\
\hline $\begin{array}{l}\text { Lamproie marine } \\
\text { (Petromyzon marinus) }\end{array}$ & 74 & 98 & 112 & 83 & 105 & 107 & 124 & 90.2 & 41 & 75.3 & 152.5 & 40.5 & 48 & 45.7 \\
\hline $\begin{array}{l}\text { Anguille civelle } \\
\text { (Anguilla anguilla) }\end{array}$ & 190 & 332 & 123 & 80 & 82 & 33 & 80 & 48 & 64 & 42 & 69 & 46 & 73.2 & 30 \\
\hline $\begin{array}{l}\text { Anguille subadulte } \\
\text { (Anguilla anguilla) }\end{array}$ & 400 & 336 & 291 & 148 & 267 & 206 & 107 & 78.5 & 42 & 73.7 & 57 & 56.3 & 55.5 & 33.5 \\
\hline $\begin{array}{l}\text { Alose feinte } \\
\text { (Alosa fallax) }\end{array}$ & 127 & $?$ & $?$ & $?$ & $?$ & 75 & & & & & & & & \\
\hline Autres espèces & $200^{*}$ & $500^{*}$ & $500^{*}$ & $500^{*}$ & $400^{*}$ & $?$ & & & & & & & & \\
\hline
\end{tabular}

\section{Situation des aloses}

La reconquête des bassins versants par la grande alose a été la plus spectaculaire. Des spécimens sont fréquemment observés à plus de $300 \mathrm{~km}$ de l'estuaire, sur la Garonne en amont de Toulouse et sur la Dordogne dans le département de la Corrèze. Au niveau des stations de contrôle, l'augmentation des effectifs entre 1991 et 1997 illustre cette colonisation massive des cours d'eau nouvellement ouverts à la libre circulation des aloses. En revanche, les données de capture dans l'estuaire de la Gironde ne font pas apparaître d'évolution évidente de la population de grandes aloses. Depuis 1998, les stations de contrôle montrent d'importantes fluctuations inter-annuelles des migrations. Ce phénomène est à relier aux conditions hydroclimatiques. Par ailleurs, le suivi des frayères sur la Garonne a montré une suractivité de frai en aval de Golfech les années où les plus faibles effectifs ont été enregistrés sur cette station (BELAUD et CARETTE, 1999).

La situation de l'alose feinte est assez imprécise. En effet, les habitats de reproduction de l'espèce sont situés en aval des premières stations de vidéo-contrôle (Golfech et Tuilières), et la faible exploitation commerciale de l'espèce ne permet pas de disposer de données précises à travers le suivi des pêcheries.

\section{Situation de l'anguille}

Concernant l'anguille, les indicateurs halieutiques de l'estuaire confirment la tendance observée au niveau national et international d'un effondrement des stocks dans les années quatre-vingt. Sur les dernières années, des fluctuations sont constatées avec une tendance de poursuite à la baisse. Au niveau des stations de contrôle, les chiffres enregistrés ne sont pas représentatifs de l'abondance de l'espèce sur les bassins car indépendamment des systèmes de comptage qui ne permettent pas une bonne visualisation des individus migrants, les dispositifs de franchissement ne sont pas adaptés aux anguillettes. Aucune donnée ne peut ainsi être rationnellement exploitée pour ces stations. 


\section{Situation des lamproies}

Tout comme la grande alose, la lamproie marine est désormais fréquemment observée dans les parties amont des bassins. Sur la Dordogne, sa présence est rapportée jusqu'au pied de la plupart des premiers barrages infranchissables. Les passages enregistrés au niveau des stations de contrôle montrent une nette différence de répartition de la population entre le bassin de la Garonne et celui de la Dordogne. Alors que sur la Dordogne, les effectifs contrôlés à Tuilières semblent se stabiliser depuis 1994 entre 3000 et 4500 individus, sur la Garonne les effectifs sont beaucoup plus faibles et les variations inter-annuelles nettement plus marquées. Sur cet axe, les résultats du suivi peuvent varier d'un facteur 20 selon les années et la colonisation du bassin amont est moins importante : seules une centaine d'individus par an sont dénombrés en moyenne au Bazacle.

Tout comme pour l'alose feinte, les connaissances sur la population de lamproies fluviatiles se borne aux observations ponctuelles rapportées par les pêcheurs. Celles-ci indiquent une très importante diminution des stocks au cours des dernières décennies.

\section{Situation des salmonidés}

La pêche des salmonidés étant strictement interdite, le contexte réglementaire est défavorable à la déclaration de captures accidentelles. Aussi on ne dispose d'aucune donnée fiable sur la situation de ces espèces dans l'estuaire de la Gironde, dans la basse Dordogne et dans la basse Garonne. Au niveau des stations de contrôle, on constate globalement une augmentation des effectifs à partir des années 1993 et 1994 avec toutefois des fluctuations interannuelles. Sur la Dordogne et sur la Garonne, on peut actuellement quantifier le stock des géniteurs susceptibles de participer à la reproduction dans la mesure où toutes les zones de frayères accessibles sont situées en amont de ces points de contrôle. II subsiste toutefois des incertitudes sur la part de ce stock qui atteint réellement les zones de reproduction et sur l'efficacité de celle-ci, notamment sur des axes soumis à l'exploitation hydroélectrique par éclusées (Dordogne, Maronne, Cère, Vézère, Garonne amont). Un suivi des frayères engagé depuis quelques années devrait permettre de lever une partie de ces incertitudes. Pour autant, ce suivi ne permet pas de discriminer les frayères à truites de mer des frayères à saumons. Par ailleurs, une étude engagée par E.P.I.DOR. en vue de limiter des effets des éclusées sur le bassin versant de la Dordogne (ANONYME, 1999) doit conduire à fixer des conditions d'exploitation des barrages hydroélectriques compatibles avec la vie des saumons. Une incertitude concerne la part de géniteurs qui resterait bloquée en dessous des barrages, les années où les conditions thermiques et hydrologiques sont défavorables. Sur la Garonne, certaines années, seulement la moitié des effectifs de saumons recensés à la station de Golfech atteint la station du Bazacle à Toulouse. Ceci semble dû à la gestion de l'ascenseur à poissons de Golfech, qui peut induire un retard à la migration, et peut-être également aux caractéristiques des souches utilisées qui semblent induire un déséquilibre en faveur des migrations de fin de printemps, de début d'été et d'automné. Par ailleurs la qualité des informations obtenues est très inégale introduisant vraisemblablement un biais d'une station à l'autre en raison de qualités d'image vidéo variables.

\section{Situation de l'esturgeon}

Le stock demeure à un très faible niveau d'abondance. De 1981 à 1997, seulement 37 adultes migrants sont signalés sur la Gironde, la Garonne et la Dordogne et les dernières reproductions naturelles avérées sont enregistrées en 1994 et 1988 (ROCHARD et al., 1997 b). Une reproduction artificielle réalisée en 1995 a permis de déverser environ 9000 alevins dans le milieu naturel, à trois stades différents (WILLIOT, 1997 d; BRUN et ROUAULT, 1997 b ; BRUN et ROUAULT, 1997 c ; ANONYME, 1997). Aujourd'hui, le devenir de la population repose essentiellement sur l'avenir de ces cohortes dont les premiers retours sont attendus aux environs de l'année 2000. 


\section{DISCUSSION}

Les techniques de restauration des poissons migrateurs ont très largement progressé. On peut toutefois relever des manques dans plusieurs domaines. Sur les aspects réglementaires, on observe par exemple un important déséquilibre entre les mesures prises en faveur de la protection des salmonidés, pour lesquels la réglementation est très complète et celles prises pour les autres espèces, notamment pour l'anguille. On note également un défaut d'harmonisation entre les mesures de protection des biotopes et les réglementations sur le classement des cours d'eau au titre de la libre circulation, certains cours d'eau étant par exemple classés pour quatre espèces alors que seulement deux d'entre-elles sont concernées au titre de la protection des biotopes. Cette situation, qui apparaissait plutôt cohérente dans une phase de reconquête pour laquelle les entraves à la migration constituaient le principal facteur limitant, ne favorise pas la meilleure prise en compte de la qualité des habitats aujourd'hui rendus accessibles.

On a pu constater que les études de potentiel réalisées sur la plupart des cours d'eau ont été très ciblées, en vue notamment d'opérations de repeuplement pour le saumon, alors que les reconnaissances réalisées sur ces cours d'eau auraient pu être valorisées pour les autres espèces. Au début des programmes, les travaux réalisés pour améliorer la libre circulation ont été très ciblés sur les salmonidés. Ils ont ensuite intégré les besoins de l'alose. Aucun effort spécifique n'a concerné l'anguille avant la mise en place, en 1997, d'une expérimentation de passe à anguillettes / civelles sur la Dordogne au barrage de Tuilières. Ainsi aucune démarche intégrant des objectifs globaux, communs à l'ensemble des espèces n'a réellement été mise en place.

Le facteur limitant " barrage » a constitué quasiment l'essentiel du programme de restauration, mobilisant $70 \%$ des coûts du programme, de telle sorte que, dès le départ, d'autres aspects techniques ont été minimisés ou sous évalués. Ainsi sur la Garonne le potentiel de production initialement évalué à environ 450000 équivalents-smolts sauvages (soit une production théorique de 8 smolts par $100 \mathrm{~m}^{2}$ ) a du être revu à la baisse et les connaissances actuelles ont conduit à réduire de $2 / 3$ l'évaluation du potentiel productif (EUZENAT et PORCHER, 1999). Sur le thème de la libre circulation, il faut également noter la prise en compte très récente du problème de la dévalaison dans les stratégies de réouverture des cours d'eau pour la libre circulation des poissons migrateurs. Les connaissances en la matière sont encore récentes. Certaines solutions techniques ont été mises au point notamment pour le saumon. Pour les autres espèces, il est encore nécessaire d'acquérir des connaissances fondamentales sur le comportement des poissons face à l'ouvrage.

En matière de suivi des populations, l'ensemble du système Gironde-GaronneDordogne dispose de deux outils structurants avec d'une part le suivi halieutique de l'estuaire de la Gironde et d'autre part les stations de vidéo-contrôle des migrations en place sur la Dordogne et sur la Garonne. Ces outils permettent de disposer de données essentielles pour la connaissance des populations mais ils sont insuffisants pour les apprécier de façon complète. En premier lieu, ces outils ne présentent pas la même fiabilité pour toutes les espèces. En effet, le suivi halieutique dans sa forme actuelle, ne fournit une image que pour les espèces commercialement exploitées. II n'apporte par exemple aucun élément sur la situation des salmonidés. Les stations de contrôle de Tuilières sur la Dordogne et de Golfech sur la Garonne sont quant à elles, conditionnées par le fonctionnement des ascenseurs à poissons. Ces dispositifs, n'étant pas adaptés à l'anguille, fournissent des informations biaisées sur cette espèce. La position de ces stations de contrôle dans le bassin versant est également un élément important. Les stations de Tuilières et de Golfech, étant situées dans la partie moyenne des axes Dordogne et Garonne, permettent de contrôler la totalité des géniteurs migrants vers leurs zones de frayères pour les salmonidés, mais seulement une partie d'entre eux pour la 
grande alose et les lamproies, qui disposent d'habitats de reproduction en aval. Les données de migration de ces espèces doivent donc être interprétées en fonction du comportement de migration, qui d'une année sur l'autre peut être différent, à cause des variations environnementales inter-annuelles. Pour fournir une image fiable des populations, des moyens complémentaires devront donc être mis en place, et ce, pour chaque espèce.

Le suivi du milieu, qui par endroit (notamment sur la Garonne amont) s'avère très dégradé tant au niveau de la qualité de l'eau et des habitats que de la quantité d'eau (valeur et respect des débits réservés) nª pas été intégré au dispositif d'évaluation. II apparaît de façon évidente que si chaque opérateur a tenté d'évaluer ses propres actions, il y a fondamentalement un fort déficit de coordination de cette évaluation. Cette lacune rend le suivi de la partie biologique des programmes (habitats, efficacité des repeuplements) particulièrement délicat. L'évaluation du programme dans son ensemble s'impose de toute urgence (EUZENAT et PORCHER, 1999). II faut véritablement mettre en place des moyens d'évaluation permettant d'une part de mesurer chaque année l'avancement du projet, et d'autre part de fournir des informations utiles à la prise de décisions, éventuellement à la réorientation du programme (BAGLINIERE, 1998). Cette réflexion est actuellement en cours sur les deux bassins.

\section{BIBLIOGRAPHIE}

ANONYME, 1992 a. Evaluation du potentiel salmonicole de la rivière Vézère en aval du barrage du Saillant - Départements de la Corrèze et de la Dordogne. Association Connaissance de la Vie Fluviale, $78 \mathrm{p}$.

ANONYME, 1992 b. Evaluation du potentiel salmonicole de la rivière Corrèze. Département de la Corrèze. Association Connaissance de la Vie Fluviale, $37 \mathrm{p}$.

ANONYME, 1997. Restauration de l'esturgeon européen (Acipenser sturio). Contrat Life $n^{\circ}$ B4-3200/94/754, rapport final. Programme LIFE 94, E.P.I.DOR., 26 p.

ANONYME, 1998 a. Sauvegarde et restauration de l'esturgeon européen. Programme d'exécution LIFE-Nature 98. Rapport E.P.I.DOR.- CEMAGREF Bordeaux.

ANONYME, 1998 b. Repeuplements sur le bassin de la Dordogne, saumon atlantique. Rapport Conseil Supérieur de la Pêche-DR7, $21 \mathrm{p}$.

ANONYME, 1999. Etude des éclusées des axes Dordogne, Cère, Maronne, Vézère. Premiers diagnostics. Rapport intermédiaire. E.P.I.DOR., Compagnie des Experts et Sapiteurs, $65 \mathrm{p}$.

BAGLINIERES J.L., 1998. Avis du GRISAM sur le dossier « Objectif 2003 : propositions pour une stratégie de gestion et de repeuplement du saumon atlantique dans les cours d'eau du bassin de la Dordogne ". GRISAM, 6 p.

BELAUD A., CARETTE A., 1999. Suivi 1999 de la qualité des milieux et de la reproduction des aloses à Agen et en moyenne Garonne. Rapport ENSAT, $61 \mathrm{p}$.

BRUN R., ROUAULT T., 1997 a. Détermination des conditions optimales de capture et de transfert, mise au point et mise en cuvre des procédures de récupération des poissons. Restauration de l'esturgeon européen (Acipenser sturio). Contrat Life $n^{\circ}$ B4-3200/94/754, rapport final du programme d'exécution. Opération 1 - II - III. Programme LIFE 94, étude CEMAGREF - Bordeaux, $n^{\circ} 24,1-18$.

BRUN R., ROUAULT T., 1997 b. Définition des conditions minimales pour le lâcher des juvéniles. Restauration de l'esturgeon européen (Acipenser sturio). Contrat Life $\mathrm{n}^{\circ} \mathrm{B} 4-3200 / 94 / 754$, rapport final du programme d'exécution. Opération I - II - III. Programme LIFE 94, étude CEMAGREF - Bordeaux, $n^{\circ}$ 24, 93-108. 
BRUN R., ROUAULT T., 1997 c. Définition des modalités de lâcher des juvéniles dans le milieu naturel. Restauration de l'esturgeon européen (Acipenser sturio). Contrat Life $n^{\circ}$ B4-3200/94/754, rapport final du programme d'exécution. Opération I - II - III. Programme LIFE 94, étude CEMAGREF - Bordeaux, $n^{\circ} 24,109-117$.

BRUZY A., 1985. Saumon atlantique. Possibilités de réimplantation sur le Viaur, $46 \mathrm{p}$.

CARRY L., BOYER-BERNARD S., 1999. Etude des rythmes de migration des espèces amphibiotiques et holobiotiques de la Garonne au niveau de la station de contrôle de Golfech au cours de l'année 1998. Rapport MI.GA.DO. G6-99-RT, 26 p.

CASSOU-LEINS F., CASSOU-LEINS J.J., DAUBA F., LEJOLIVET C., 1988. Réserve naturelle de la frayère d'alose d'Agen. Etude de l'alevin d'alose (Alosa alosa L.). Répartition, croissance, régime alimentaire, $24 \mathrm{p}$.

CASSOU-LEINS F., CASSOU-LEINS J.J., 1981. Recherches sur la biologie et I'halieutique des migrateurs de la Garonne et principalement l'alose (Alosa alosa L.). Thèse doctorat de troisième cycle, Institut National Polytechnique de Toulouse, $382 \mathrm{p}$.

CASTELNAUD G., LAMBERT P., ROCHARD E., 1998. Surveillance halieutique de l'estuaire de la Gironde. Suivi statistique 1996, étude de la faune circulante 1997. Etude CEMAGREF - Bordeaux $n^{\circ}$ 97. EDF CPN Blayais, $153 \mathrm{p}$.

CAUDRON A., CHEVRE P., 1999. Suivi de la reproduction naturelle du saumon atlantique (Salmo salar L.) sur le bassin de la Dordogne dans le département de la Corrèze durant l'hiver 98-99 et cartographie des zones de frai. Rapport MI.GA.DO, 16 p.

CHEVRE P., 1997. Bilan des repeuplements de saumons sur le bassin de la Dordogne. Propositions d'action. Rapport MI.GA.DO., $28 \mathrm{p}$.

CHEVRE P., 1998. Pêches électriques de contrôle sur le bassin de la Dordogne (campagne 1997). MI.GA.DO., 19 p. + annexes.

CROZE O., LALLEMANT A., BOSC S., CARRY L., LARINIER M., 1998. Expérimentations sur la mise en place et l'efficacité de dispositifs de piégeage de smolts de saumon atlantique en dévalaison. Usine hydroélectrique de Pointis et Camon (Garonne 31). Rapport GHAAPPE RA98.05, 40 p. + annexes.

DARTIGUELONGUE J., 1992. Possibilité de restauration du saumon atlantique dans le bassin de la Garonne - Cartographie de la rivière Salat. Rapport SCEA-MI.GA.DO.

DARTIGUELONGUE J., 1999. Suivi de la fraie des salmonidés migrateurs sur l'Ariège et la Garonne à l'automne 1988. Rapport MI.GA.DO., 25 p.

DARTIGUELONGUE J., LANGON M., 1999. Contrôle du fonctionnement de l'ascenseur à poissons de Tuilières en 1998. Suivi de l'activité ichtyologique. Rapport MI.GA.DO. G7-99-RT, $26 \mathrm{p}$.

DULUDE P., BACH J.M., BRUGEL C., 1992. Etude de la reproduction des saumons atlantiques (Salmo salar) dans la rivière Dordogne en aval du barrage d'Argentat. Département de la Corrèze (19). Rapport Ministère de l'Environnement- CSP. MI.GA.DO., 49 p. + annexes.

DULUDE P., GUERRI O., PUSTELNIK G., CHEVRE P., 1997. Objectif 2003 : propositions pour une stratégie de gestion et de repeuplement du saumon atlantique dans les cours d'eau du bassin de la Dordogne. Rapport E.PI.DOR-MI.GA.DO., 34 p.

ELIE P., ROCHARD E., TAVERNY C., 1997. Etat des frayères-Synthèse des connaissances. Restauration de l'esturgeon européen (Acipenser sturio). Contrat Life $n^{\circ}$ B4-3200/94/754, rapport final du programme d'exécution. Opération I - II - III. Programme LIFE 94, étude CEMAGREF - Bordeaux, $n^{\circ}$ 24, 127-171.

EUZENAT G.. PORCHER J.P., 1999. Restauration du saumon dans la Garonne. Rapport de la visite d'expertise des 9 et 10 septembre 1998.

FATIN D., DARTIGUELONGUE J., 1995. Etude préliminaire de la reproduction des aloses en 1995 entre Tuilières et Mauzac sur la Dordogne. EDF - SCEA, 39 p. + annexes. 
GAYOU F., 1986. Possibilités de restauration du saumon atlantique et de l'alose dans le bassin de la Garonne (Ariège, Garonne, Aveyron, Viaur). Rapport de synthèse CSP-DR7, $87 \mathrm{p}$.

GAYOU F., 1993. Etude des potentialités de l'Hers Vif. Cartographie. Rapport MI.GA.DO.CSP DR7, $137 \mathrm{p}$.

GAYOU F., 1999. Saumon atlantique : Objectif Garonne Ariège. Bilan des actions réalisées et perspectives. Rapport de synthèse CSP-DR7, 46 p. + annexes.

GUERRI O., PUSTELNIK G., 1995. La disparition de l'esturgeon européen (Acipenser sturio) dans les bassins de la Garonne et de la Dordogne. Les mémoires d'un garde pêche. Programme LIFE 94, E.P.I.DOR., 48 p.

GUERRI O., PUSTELNIK G., 1996. Etude préliminaire sur les frayères à esturgeon européen (Acipenser sturio) sur la Garonne et la Dordogne. Programme LIFE 94, E.P.I.DOR., CEMAGREF, 32 p. + annexes.

GUIDET A., 1994. Note sur le potentiel salmonicole de la Maronne, $6 \mathrm{p}$.

JEGO S., GAZEAU C., ROCHARD E., ELIE P., 1997. Etat actuel des zones de frayères Proposition d'actions de protection et de réhabilitation. Restauration de l'esturgeon européen (Acipenser sturio). Contrat Life $n^{\circ}$ B4-3200/94/754, rapport final du programme d'exécution. Opération I - II - III. Programme LIFE 94, étude CEMAGREF - Bordeaux, $n^{\circ} 24,173-269$.

KERHOAS J., 1984. Saumon atlantique. Possibilités de réimplantation sur l'Aveyron. Rapport ISIM-DDAF Aveyron, $53 \mathrm{p}$.

LARINIER M., TRAVADE F., 1992. La conception des dispositifs de franchissement pour les aloses. Bull. Fr. Pêche Piscic. (1992), 326-327: 125-133.

LARINIER M., 1993. L'aménagement des obstacles pour la migration. Actes du colloque Pour un retour des poissons migrateurs, Adour, Charente, Dordogne, Garonne. Agence de l'Eau Adour-Garonne (1993), 191 p.

LEPAGE M., 1992 a. Analyse de la structure démographique de la population d'Acipenser sturio dans l'estuaire de la Gironde. AGEDRA, $30 \mathrm{p}$.

LEPAGE M., 1992 b. Répartition spatiale des populations d'Acipenser sturio dans l'estuaire de la Gironde. AGEDRA, 6 p.

LEPAGE M., LAMBERT P., ROCHARD E., 1993. Application de la méthode d'estimation de l'âge à l'analyse démographique de la population d'esturgeon (Acipenser sturio) dans l'estuaire de la Gironde. AGEDRA, $27 \mathrm{p}$.

MARCHAT F., DULUDE P., PUSTELNIK G., ROQUEPLO C., 1994. Etude de l'impact du suréquipement du barrage du Sablier sur la reproduction du saumon atlantique dans la Dordogne. Association Connaissance de la Vie Fluviale, 81 p. + annexes.

MARTINEZ G., SEUBE B., CARRY L., 1999. Suivi des migrations et de la reproduction de la grande alose (Alosa alosa) sur le Tarn avec suivi de la qualité du milieu. Rapport MI.GA.DO. G5-99-RT, 28 p.

MENCHI O., MAURIES O., 1999. Contrôle de la migration des migrateurs sur l'axe TarnAveyron et étude de la qualité de l'eau sur le bas Aveyron. Rapport MI.GA.DO., $26 \mathrm{p}$.

PORCHER J.P., 1992 a. Les passes à anguilles. Bull. Fr. Pêche Piscic. (1992), 326-327 : 134-142.

PORCHER J.P., 1992 b. Poissons migrateurs - Contrat « retour aux sources ". Proposition d'actions. Rapport CSP - Ministère de l'Environnement, $169 \mathrm{p}$.

PUSTELNIK G., 1982. Les saumons de la rivière Dordogne, 1189 - 1981 Ministère de l'Environnement, Direction de la protection de la nature - Fédération Départementale de la Pêche de la Dordogne, $54 \mathrm{p}$. 
ROCHARD E., WILLIOT P., CASTELNAUD G., LEPAGE M., 1991. Eléments de systématique et de biologie des populations sauvages d'esturgeons. CEMAGREF Publ., (Ed.) Acipenser. 475-507.

ROCHARD E., JATTEAU P., LEPAGE M., 1992. Mise au point d'une méthode de détermination de l'âge individuel de l'esturgeon européen (A. sturio). Association Girondine pour l'Expérimentation et le Développement des Ressources Aquatiques, $20 \mathrm{p}$.

ROCHARD E., LEPAGE M., GAZEAU C., 1997 a. Visualisation des zones estuariennes fréquentées par les juvéniles d'esturgeons, compréhension des migrations. Restauration de l'esturgeon européen (Acipenser sturio). Contrat Life $\mathrm{n}^{\circ} \mathrm{B} 4$ 3200/94/754, rapport final du programme d'exécution. Opération 1 - II - III. Programme LIFE 94, étude CEMAGREF - Bordeaux, $n^{\circ} 24,293-348$.

ROCHARD E., LEPAGE M., GAZEAU C., LAMBERT P., 1997 b. Tableau de bord de la population. Estimation de l'abondance des différentes classes d'âge. Restauration de l'esturgeon européen (Acipenser sturio). Contrat Life $n^{\circ}$ B4-3200/94/754, rapport final du programme d'exécution. Opération I - II - III. Programme LIFE 94, étude CEMAGREF - Bordeaux, $n^{\circ} 24,349-381$.

ROGUET M., 1993. Bilan du programme de restauration des poissons migrateurs dans le bassin Adour-Garonne. Actes du colloque : pour un retour des poissons migrateurs, Adour, Charente, Dordogne, Garonne. Agence de l'Eau Adour Garonne (1993), $191 \mathrm{p}$.

ROUAULT T., BRUN R., 1997. Condition d'acclimatation à court terme (0 à 6 mois) de juvéniles aux conditions d'élevage et acclimatation à long terme. Restauration de l'esturgeon européen (Acipenser sturio). Contrat Life $n^{\circ}$ B4-3200/94/754, rapport final du programme d'exécution. Opération I - II - III. Programme LIFE 94, étude CEMAGREF - Bordeaux, $n^{\circ} 24,19-44$.

SOULET A., 1994. Etude hydromorphologique de la Bave. Evaluation de son potentiel de production en saumon atlantique. Conseil Supérieur de la Pêche, $34 \mathrm{p}$.

TINEL C., 1983. Eléments pour la réintroduction du saumon atlantique dans la rivière Dordogne. Conseil Supérieur de la Pêche, pisciculture de Castels (Dordogne), 69 p.

TRAVADE F., LARINIER M., TRIVELLATO D., DARTIGUELONGUE J., 1992. Conception d'un ascenseur à poissons adapté à l'alose (Alosa alosa) sur un grand cours d'eau : l'ascenseur de Golfech sur la Garonne. Hydroécologie Appliquée, Tome 4, vol 1: 91-119.

TRAVADE F., LARINIER M., BOYER-BERNARD S., DARTIGUELONGUE J., 1996. Feedback on four fishpass installations recently built on two rivers in southwest France.

WILLIOT P., 1997 a. Préparation finale des prégéniteurs. Restauration de l'esturgeon européen (Acipenser sturio). Contrat Life $n^{\circ}$ B4-3200/94/754, rapport final du programme d'exécution. Opération I - II - III. Programme LIFE 94, étude CEMAGREF - Bordeaux, $n^{\circ} 24,45-50$.

WILLIOT P., 1997 b. Optimisation finale des procédures de reproduction des femelles. Restauration de l'esturgeon européen (Acipenser sturio). Contrat Life $n^{\circ} \mathrm{B} 4-$ 3200/94/754, rapport final du programme d'exécution. Opération I - II - III. Programme LIFE 94, étude CEMAGREF - Bordeaux, $n^{\circ}$ 24, 51-59.

WILLIOT P., 1997 c. Optimisation finale des procédures de reproduction des mâles. Restauration de l'esturgeon européen (Acipenser sturio). Contrat Life $\mathrm{n}^{\circ}$ B43200/94/754, rapport final du programme d'exécution. Opération I - II - III. Programme LIFE 94, étude CEMAGREF - Bordeaux, $n^{\circ}$ 24, 61-66.

WILLIOT P., 1997 d. Production d'alevins. Restauration de l'esturgeon européen (Acipenser sturio). Contrat Life $n^{\circ}$ B4-3200/94/754, rapport final du programme d'exécution. Opération I - II - III. Programme LIFE 94, étude CEMAGREF Bordeaux, $n^{\circ} 24,67-91$. 